\title{
Food-grade hydroxypropyl methylcellulose-based formulations for electrohydrodynamic processing: Part I - Role of solution parameters on fibre and particle production
}

\author{
P.M. Silva ${ }^{\mathrm{a}, \mathrm{d},{ }^{*}, \text { C. Prieto }^{\mathrm{b}}{ } \text {, J.M. Lagarón }}{ }^{\mathrm{b}}$, L.M. Pastrana ${ }^{\mathrm{d}}$, M.A. Coimbra ${ }^{\mathrm{c}}$, A.A. Vicente ${ }^{\mathrm{a}}$, M. \\ A. Cerqueira ${ }^{\text {d, **** }}$
}

${ }^{a}$ Centre of Biological Engineering, University of Minho, 4710-057, Braga Portugal

${ }^{\mathrm{b}}$ Novel Materials and Nanotechnology Group, IATA-CSIC, 46980, Paterna, Spain

${ }^{\mathrm{c}}$ LAQV-REQUIMTE, University of Aveiro, 3810-193, Aveiro, Portugal

d International Iberian Nanotechnology Laboratory, Av. Mestre José Veiga s/n, 4715-330, Braga, Portugal

\section{A R T I C L E I N F O}

\section{Keywords:}

Electrohydrodynamic processing

Electrospinning zone

Electrospraying zone

Aspect ratio

Specific viscosity

Nanotechnology

\begin{abstract}
A B S T R A C T
Electrohydrodynamic (EHD) processing allows the production of micro and nano structures with high surfacearea-to-volume ratio from biopolymers and environmentally friendly solvents. Such structures hold a very significant potential for application in the food area. The aim of this work was to assess the role of solution parameters in the formation of hydroxypropyl methylcellulose (HPMC)-based micro and nanostructures through EHD processing, establishing a relationship between variables such as viscosity and concentration, and processing zones (i.e., combinations of processing conditions that move the system towards electrospinning - fibres are formed - or electrospraying - particles are formed).

Micro and nano structures were produced through electrospinning and electrospraying using HPMC with low (HPMC LMW) and high (HPMC HMW) molecular weight. Solutions were characterized regarding surface tension, conductivity, viscosity, zero-shear rate and specific viscosity. Plotting specific viscosity versus concentration allowed determining the electrospraying and electrospinning zones, which were confirmed through scanning electron microscopy analysis. HPMC LMW led to the formation of particles. For concentrations between 1 and $2 \%$ $(\mathrm{w} / \mathrm{v})$ rod like particles were formed, and round particles were obtained for concentrations ranging from 3 to $6 \%$ $(\mathrm{w} / \mathrm{v})$. The mean particle diameter varied between 833 and $1188 \mathrm{~nm}$, while the aspect ratio ranged from 1.3 to 3.7. Nanofibres were generated using HPMC HMW, being beaded fibres produced at a concentration of 1\% (w/v) and smooth fibres produced for concentrations between 1.5 and $2.25 \%(\mathrm{w} / \mathrm{v})$. The developed nanofibres displayed a mean diameter ranging between 79 and $161 \mathrm{~nm}$.

Electrospraying and electrospinning zones were successfully determined for HPMC LMW and HMW. Nevertheless, near transition zones variability regarding the obtained morphology was observed once other processing parameters (e.g., flow rate) can influence the morphology of fibers and particles.
\end{abstract}

\section{Introduction}

Over the last decades, the interest in micro and nano structures has been increasing in different areas, such as food and biomedicine. Given the need for properties such as biocompatibility, low toxicity and low cost, biopolymers have been presented as one of the materials of great interest for the development of these structures (Abuzar et al., 2018; Bourbon, Barbosa-Pereira, Vicente, Cerqueira, \& Pastrana, 2020; Nunes et al., 2020; Zhang et al., 2018). The use of biopolymers in the design of micro and nano structures allows the development of structures that possess high surface-area-to-volume ratios, and a tunable and versatile morphology (Costa et al., 2019; Lasprilla-Botero, Álvarez-Láinez, \& Lagaron, 2018; Limongi et al., 2017; Senthil Muthu Kumar et al., 2019).

One of the biopolymers that has been increasingly used in the food and biomedical industries is the polysaccharide hydroxypropyl methylcellulose (HPMC). This increase is explained by its biocompatibility,

\footnotetext{
* Corresponding author. Centre of Biological Engineering, University of Minho, 4710-057, Braga, Portugal.

** Corresponding author. International Iberian Nanotechnology Laboratory, Av. Mestre José Veiga s/n, 4715-330, Braga, Portugal.

E-mail addresses: pedro.silva@ceb.uminho.pt (P.M. Silva), miguel.cerqueira@inl.int (M.A. Cerqueira).
} 
low toxicity, solubility in different solvents, and approval for use in a wide range of applications. HPMC is a cellulose ether with substituted hydroxyl groups, allowing for control of properties, such as solubility and viscosity (Burdock, 2007; Kaur et al., 2018; Pal, Paulson, \& Rousseau, 2013; Sun, Liang, Tan, \& Wang, 2018). It is currently widely used as a direct or indirect food additive in the food industry (Chowhan, 1980; Stephen, Phillips, \& Williams, 2016; Tanti, Barbut, \& Marangoni, 2016).

These industries have widely used the encapsulation of bioactive compounds or drugs through methodologies such as spray-drying, complex coacervation, emulsification and salting-out (Cerqueira et al., 2014; Karim et al., 2016; Katona, Sovilj, Petrović, \& Milanović, 2010; Romita, Cheng, \& Diosady, 2011; Silva et al., 2019). Currently used techniques have disadvantages such as making use of temperature, pressure, or leading to low encapsulation efficiencies, which can be a problem depending on the encapsulated compound and the field of application (Costa et al., 2019; García-Moreno, Mendes, Jacobsen, \& Chronakis, 2018; Marques et al., 2019; Rodrigues et al., 2020). Electrohydrodynamic (EHD) processing arises as an up-and-coming encapsulation technology, presenting a high encapsulation efficiency, low cost, allowing room or ambient working conditions, as well as controllable temperature and humidity, if needed. EHDs allows a versatile production of micro and nano structures with high surface-area-to-volume ratio, combined with a narrow size distribution, simply by fine tuning its processing parameters. These characteristics and properties are of extreme interest for the production of micro and nano structures for the food industry, as the recent increase in publications and applications in this area shows (Costa et al., 2019; Deng, Kang, Liu, Feng, \& Zhang, 2018; García-Moreno et al., 2018; Liao, Loh, Tian, Wang, \& Fane, 2018; Rodrigues et al., 2020; Senthil Muthu Kumar et al., 2019). Parameters that influence structure morphology include voltage, tip-to-collector distance, flow rate, the selected solvent and polymer, its concentration and the viscosity of the solution obtained with it (Costa et al., 2019; García-Moreno et al., 2018; Marques et al., 2019; Senthil Muthu Kumar et al., 2019; Wang, Jansen, \& Yang, 2019).

EHD processing has been used to produce particles and fibres using different polysaccharides. However, despite HPMC being frequently used in the food and biomedical industries, its stand-alone use in EHD processing for the development of food grade micro and nano structures is unexplored. Existing reports typically make use of non-aqueous and non-GRAS solvents, limiting the applicability of the developed structures based on HPMC in the food industry. In EHD processing, HPMC is most often used blended with other polymers or used to produce solid amorphous dispersions that lack a defined and reproducible morphology (Aydogdu, Sumnu, \& Sahin, 2019; Mahesh, Kathyayani, Nanjundaswamy, Channe Gowda, \& Sridhar, 2019; Smeets, Clasen, \& Van den Mooter, 2017; Smeets, Koekoekx, Clasen, \& Van den Mooter, 2018; Zhou et al., 2019). Therefore, the need exists for exploring the use of food-grade biomaterials, such as HPMC, in EHD processing technologies, with food grade and generally recognized as safe solvents, for the development of micro and nano structures with well-defined and reproducible morphology. Viscosity and surface tension are essential parameters that need to be studied in order to predict the produced structures (particles or fibres, thus defining electrospinning and electrospraying zones, respectively) and to control their morphology through EHD processing. To this point, such work has not yet been developed while using HPMC (Faramarzi, Barzin, \& Mobedi, 2016; Huang et al., 2019; Lee et al., 2018). In this sense, an analysis of the relationship between concentration, viscosity, surface tension, concentration regimes and chain entanglements is important to define the electrospinning and electrospraying zones. This kind of study has been previously used with other polymers, such as polyimide, zein, poly (lactic-co-glycolic acid), among others, but has not yet been used for HPMC (Bhushani, Kurrey, \& Anandharamakrishnan, 2017; Lasprilla-Botero et al., 2018; Tiwari \& Venkatraman, 2012).

In this work, high and low molecular weight HPMC were used to produce food-grade fibres and particles by an EHD process (electrospinning and electrospraying, respectively), using an ethanol-water mixture $(75 \%)$ as solvent. A relationship between the viscosity of the formulations and the morphology of electrospun fibres or particles was established, and potential electrospinning and electrospraying zones were proposed. Polymer solutions were then combined with electrohydrodynamic working conditions to produce micro and nano structures from HPMC. The developed micro and nano structures were then characterised regarding their morphology, and electrospinning and electrospraying zones were determined.

\section{Materials and methods}

\subsection{Materials}

Hydroxypropyl methylcellulose (HPMC 45847) (methoxyl 28-30\%, hydroxypropyl $7-12 \%$, viscosity $2 \%$ aqueous solution with a viscosity range of $7500-14000 \mathrm{mPa} \mathrm{s}$, at $20^{\circ} \mathrm{C}, 746 \mathrm{kDa}$ ) and hydroxypropyl methylcellulose (HPMC 44779) (methoxyl 28-30\%, hydroxypropyl $7-12 \%$, viscosity $2 \%$ aqueous solution with a viscosity range of $40-60$ $\mathrm{mPa}$ at $20^{\circ} \mathrm{C}, 90 \mathrm{kDa}$ ) were purchased from Alfa Aesar GmbH \& Co KG (Germany), absolute ethanol EPR PH.EUR. (>99.5\%) was purchased from LabKem (Spain).

\subsection{Preparation of polymer solutions for electrohydrodynamic processing}

$\operatorname{HPMC}(1,2,3,4,5$ and 6\% of HPMC 44779, and 1, 1.5, 1.75, 2 and $2.5 \%$ of HPMC 45847) was slowly added to a solvent consisting of a mixture of pure ethanol $(75 \% \mathrm{v} / \mathrm{v})$ and distilled water $(25 \% \mathrm{v} / \mathrm{v})$ in a closed plastic container and left to stir magnetically overnight at room temperature $\left(\approx 20{ }^{\circ} \mathrm{C}\right)$. The polymer solutions obtained were then filtered through a mesh to remove any solid impurities and were ready to be used after standing for a few hours to ensure removal of air bubbles. Ethanol at $75 \%(v / v)$ was selected as solvent due to the partial solubility of HPMC in both ethanol and water. These conditions will allow a higher versatility for the produced structures, which will be then able to incorporate both lipophilic and hydrophilic compounds.

\subsection{Characterisation of polymer solutions}

\subsubsection{Rheological analysis}

A Discovery Hybrid Rheometer (DHR) (TA Instruments, New Castle, USA) was used to determine the zero-shear rate and the specific viscosity of the solutions. TA Instruments Trios v.4.1.133073 software was used to collect the data. Up-down-up flow sweeps between $0.01 \mathrm{~s}^{-1}$ and 300 $\mathrm{s}^{-1}$ were conducted, using a $60 \mathrm{~mm}, 2.006^{\circ}$, cone geometry, at room temperature $\left(\approx 25^{\circ} \mathrm{C}\right)$.

\subsubsection{Surface tension}

Surface tension was determined using a Force Tensiometer- K20 (Kruss, Hamburg, Germany) using the Du Noüy ring method. $15 \mathrm{~mL}$ of the sample were placed in a vessel placed on the tensiometer platform, then a Du Noüy ring was suspended from the pendulum and place inside the vessel to be analysed. The Du Noüy ring was carefully cleaned, with abundance of water, between measurements. Samples were analysed at room temperature $\left(\approx 23^{\circ} \mathrm{C}\right)$.

\subsubsection{Electrical conductivity}

Solution conductivity was measured using a HI2003-02 Edge conductivity meter (Hanna, Rhode Island, USA). $5 \mathrm{~mL}$ of solution were transferred to a Falcon tube and the probe was submerged until the sensors were covered and stabilised. All measurements were made at room temperature $\left(\approx 23^{\circ} \mathrm{C}\right)$. 


\subsection{Electrohydrodynamic processing conditions}

The EHD processing equipment was a Spinbox Systems ${ }^{\circledR}$ from Bioinicia S.L. (Valencia, Spain) and was equipped with a variable high voltage power supply $(0-30 \mathrm{kV}) .10 \mathrm{~mL}$ plastic syringes were used to host the polymer solutions and were electrospun and electrosprayed under a steady flow rate using a blunt stainless-steel needle with a diameter of $0.601 \mathrm{~mm}(20 \mathrm{G})$. The needle was connected to the syringe through a Polytetrafluoroethylene (PTFE) tube and was horizontal to the collector. The syringe was coupled to a digitally controlled syringe pump. Electrohydrodynamic testing conditions are presented in Table 1. The relative humidity ranged between $31 \%$ and $51 \%$ for all experiments.

\subsection{Characterisation of structures}

\subsubsection{Morphology}

Produced micro and nano structures were analysed regarding their morphology using a scanning electron microscope (Hitachi S-4800, Tokyo, Japan) after being coated with a gold-palladium mixture under vacuum for $3 \mathrm{~min}$ (SC7640, Polaron, Kent, UK). SEM analyses were carried out with 1-2 mg of sample at $10 \mathrm{kV}$. Between 100 and 200 fibres or particles were measured using ImageJ software (v1.52a).

Samples were analysed regarding diameter, diameter distribution, aspect ratio and aspect ratio distribution for particles and diameter and diameter distribution for fibres. At least 200 fibres or particles were measured. The particle diameter is represented as the average height and length of particles.

Particle diameter distribution measures the homogeneity of the produced microstructures (Equation (1)), while the particle aspect ratio (Equation (2)) assesses structure morphology, which is required to be spherical. Particle aspect ratio distribution (Equation (3)) assesses how homogeneous the shape of the produced particles is across the diameter range of the produced particles.

Particle diameter distribution $=\left(\frac{\text { Particle diameter standard deviation }}{\text { Particle Diameter }}\right)^{2}$

Particle aspect ratio $=\frac{\text { Particle Height }}{\text { Particle Lenght }}$

Particle aspect ratio distribution $=\left(\frac{\text { Particle aspect ratio standard deviation }}{\text { Particle Aspect Ratio }}\right)$

Fibre diameter distribution (Equation (4)) measures the homogeneity of the produced nanostructures which is optimal when at its lowest.

Fibre diameter distribution $=\left(\frac{\text { Fibre Diameter standard deviation }}{\text { Fibre Diameter }}{ }^{2}{ }^{2}\right.$

\subsection{Statistical analyses}

Statistical analyses were performed using analysis of variance, Tukey's mean comparison test $(p<0.05)$ and results were reported as an average and standard deviation, using Origin 9.0 software (OriginLab Corporation, 2012) and GraphPad Prism 8.4.3 (GraphPad Software, LLC, 2020). A minimum of 3 replicates were performed.

\section{Results and discussion}

\subsection{Solution properties}

The selection of a solvent is intertwined with the conductivity and surface tension, as these two properties can vary significantly from solvent to solvent. In EHD processing, a higher conductivity is usually associated with smoother and smaller micro and nano structures, as it allows an easier formation of the Taylor cone, needed for the correct formation of the fibres and particles. Surface tension is another critical parameter in the formation of the Taylor cone: a lower surface tension results in easier EHD processing. The applied voltage must overcome the solvents' surface tension in order to develop a Taylor cone. In this case, the use of an ethanol/water mixture $(75 \% \mathrm{v} / \mathrm{v})$ leads to a decrease of water surface tension, from $57.17 \mathrm{mN} / \mathrm{m}$ for water to around $25 \mathrm{mN} / \mathrm{m}$ for the ethanol/water mixture. The same behaviour was observed for polymer solutions. The conductivity, on the other hand, increased from $0.37 \mu \mathrm{S} / \mathrm{cm}$ for distilled water to $0.9 \mu \mathrm{S} / \mathrm{cm}$ for the ethanol/water mixture, as shown in Table 2 . The biopolymer also affects the conductivity, where higher concentrations and MW lead to higher conductivity values. Results showed that HPMC solutions with the same polymer concentration ( 1 and $2 \% \mathrm{w} / \mathrm{w}$ from both HP) but different MW (low and high), had different conductivity results, with solutions prepared from HPMC with higher MW displaying higher values of conductivity.

Results also show that the polymer concentration and MW influence viscosity. Typically, higher MWs and polymer concentrations result in higher viscosity values for the polymer solutions, which then affect the production of micro and nano structures. This influence is explained by the increase in chain entanglements that occurs for higher MW polymers and at higher concentrations. Chain entanglements represent the physical interlocking of polymer chains, and for polymer solutions the solution entanglement number $\left(n e_{(s o l n)}\right)$ is also affected by the polymer concentration. Higher chain entanglements help stabilising the electrospinning jet, allowing for solvent evaporation and fibre formation (McKee, Wilkes, Colby, \& Long, 2004; Shenoy, Bates, Frisch, \& Wnek, 2005)

The magnitude of $n e_{(s o l n)}$ values obtained will depend on the regime of the solution. These regimes are divided in: i) "dilute regime", typically more appropriate for electrospraying $\left(n_{\mathrm{e}}\right)_{\text {soln }}<2$; ii) "semi-dilute unentangled regime" for $n_{e(s o l n)}$ values between 2 and 3.5, where the likelihood of electrospinning of both fibres and beads increases, and iii) "semi-dilute entangled regime" obtained for $n_{e(s o l n)}$ values higher than 3.5 , where electrospinning of smooth fibres is more likely to happen (Bock, Dargaville, \& Woodruff, 2012; Lee et al., 2018; McKee et al., 2004; Shenoy et al., 2005).

Thus, the viscosity and concentration of polymer solutions and MW of the polymer are extremely important in the prediction of the type of structure that can be obtained. Supplier information indicates that HPMC 44779 is of low viscosity (40-60 mPa s in a $2 \%$ aqueous solution at $20{ }^{\circ} \mathrm{C}$ ), which can be adequate for electrospraying, while HPMC 45847 has a higher viscosity $(7500-14000 \mathrm{mPa} s$ in a $2 \%$ aqueous solution at $20{ }^{\circ} \mathrm{C}$ ), and thus could be used to produce nanofibers (Lasprilla-Botero et al., 2018; Tiwari \& Venkatraman, 2012).

Fig. 1 presents the rheological profile of the formulations. The polymer solutions displayed a non-Newtonian behaviour, within the concentration range tested. The viscosity decreased for higher shear rate, being this trend more noticeable in the HPMC HMW samples. Fig. 1 shows the dependence of viscosity on concentration, with higher concentrations leading to higher viscosity values. For a proper comparison

Table 1

Electrohydrodynamic processing testing conditions.

\begin{tabular}{|c|c|c|c|c|c|c|}
\hline Biopolymer & Solvent & Needle & Tip-to-collector distance $(\mathrm{cm})$ & Voltage (kV) & Concentration (w/v \%) & Flow rate $(\mathrm{mL} / \mathrm{h})$ \\
\hline Hydroxypropyl methylcellulose LMW & & & 15 & 10 & 1 to 6 & 0.5 to 1 \\
\hline Hydroxypropyl methylcellulose HMW & Ethanol 75\% (v/v) & $20 \mathrm{G}(0.601 \mathrm{~mm})$ & 20 & 12 to 18 & 1 to 2.25 & 1 \\
\hline
\end{tabular}


Table 2

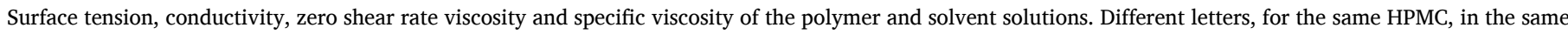
column indicate statistically significant differences $(\mathrm{p}<0.05)$.

\begin{tabular}{|c|c|c|c|c|c|}
\hline Sample & Concentration $(\mathrm{w} / \mathrm{v})$ & Surface tension $(\mathrm{mN} / \mathrm{m})$ & Conductivity $(\mu \mathrm{S} / \mathrm{cm})$ & Zero shear rate viscosity $(\mathrm{mPa} \cdot \mathrm{s})$ & Specific viscosity \\
\hline Water & - & $57.2 \pm 0.8$ & $0.37 \pm 0.12$ & N.D. & N.D. \\
\hline Ethanol & $75 \%(\mathrm{v} / \mathrm{v})$ & $25.1 \pm 0.1$ & $0.90 \pm 0.00$ & N.D. & N.D. \\
\hline \multirow[t]{6}{*}{ HPMC LMW } & 1 & $25.0 \pm 0.1^{\mathrm{a}}$ & $5.13 \pm 0.06^{\mathrm{a}}$ & $15.71 \pm 12.40^{\mathrm{a}}$ & $8.76 \pm 7.71^{\mathrm{a}}$ \\
\hline & 2 & $25.0 \pm 0.1^{\mathrm{a}}$ & $9.50 \pm 0.20^{\mathrm{b}}$ & $111.93 \pm 51.65^{\mathrm{a}}$ & $68.57 \pm 32.11^{\mathrm{a}}$ \\
\hline & 3 & $25.3 \pm 0.1^{\mathrm{b}}$ & $13.00 \pm 0.10^{c}$ & $340.29 \pm 25.04^{\mathrm{a}}$ & $210.51 \pm 15.56^{\mathrm{a}}$ \\
\hline & 4 & $25.1 \pm 0.1^{\mathrm{ab}}$ & $16.73 \pm 0.25^{\mathrm{d}}$ & $769.20 \pm 64.06^{\mathrm{b}}$ & $477.11 \pm 39.82^{\mathrm{b}}$ \\
\hline & 5 & $25.1 \pm 0.1^{\mathrm{ab}}$ & $19.40 \pm 0.40^{\mathrm{e}}$ & $1633.86 \pm 93.96^{c}$ & $1014.54 \pm 58.40^{c}$ \\
\hline & 6 & $25.1 \pm 0.1^{\mathrm{ab}}$ & $22.33 \pm 0.12^{\mathrm{f}}$ & $3509.49 \pm 382.28^{\mathrm{d}}$ & $2180.36 \pm 194.01^{d}$ \\
\hline \multirow[t]{5}{*}{ HPMC HMW } & 1 & $24.9 \pm 0.1^{\mathrm{a}}$ & $8.33 \pm 0.06^{\mathrm{a}}$ & $1030.73 \pm 95.35^{\mathrm{a}}$ & $639.66 \pm 59.26^{\mathrm{a}}$ \\
\hline & 1.5 & $25.1 \pm 0.1^{\mathrm{ab}}$ & $12.30 \pm 0.27^{\mathrm{b}}$ & $6687.30 \pm 774.62^{\mathrm{b}}$ & $4155.56 \pm 481.47^{\mathrm{b}}$ \\
\hline & 1.75 & $25.3 \pm 0.1^{\mathrm{b}}$ & $13.87 \pm 0.06^{\mathrm{c}}$ & $11656.11 \pm 2420.33^{c}$ & $7243.97 \pm 1504.38^{c}$ \\
\hline & 2 & $25.7 \pm 0.1^{\mathrm{c}}$ & $15.97 \pm 0.15^{\mathrm{d}}$ & $19312.61 \pm 3181.63^{d}$ & $12002.95 \pm 1440.03^{d}$ \\
\hline & 2.5 & $26.1 \pm 0.1^{\mathrm{d}}$ & $17.23 \pm 0.23^{\mathrm{e}}$ & $33035.88 \pm 1012.30^{\mathrm{e}}$ & $20090.98 \pm 924.10^{\mathrm{e}}$ \\
\hline
\end{tabular}
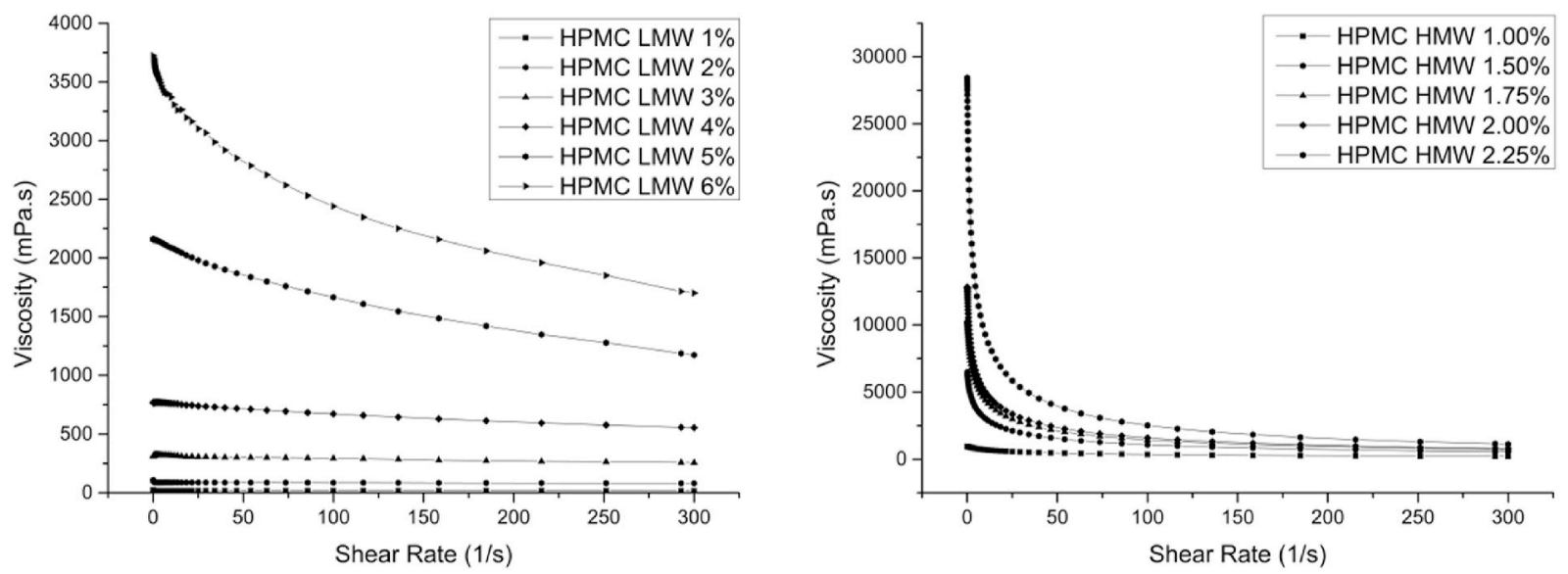

Fig. 1. Viscosity profile of polymer solutions over a range of shear rates $\left(0.01-300 \mathrm{~s}^{-1}\right)$.

between samples, the zero-shear rate viscosity $\left(\eta_{0}\right)$ of each sample was determined through data fitting by Cross's model (Stephen et al., 2016), and is presented in Table 2. Results show that only at its higher concentrations ( $>4 \%$ ) the HPMC LMW present values close to the HPMC HMW values at the lowest concentration (1\%).

Table 2 shows that the zero-shear rate viscosity increases from 15.71 $\mathrm{mPa} s$ to $3509.49 \mathrm{mPa} s$ for the HPMC LMW, as concentration increases from 1 to $6 \%(\mathrm{w} / \mathrm{v})$, while for the HPMC HMW viscosity increases from $1030.73 \mathrm{mPa} s$ to $33035.88 \mathrm{mPa} \mathrm{s}$ when the concentration increases from 1 to $2.25 \%(\mathrm{w} / \mathrm{v})$. The influence of the polymer on the $\eta_{0}$ was evaluated by determining each solution's specific viscosity $\left(\eta_{s p}\right)$, through Equation 5, in which $\eta_{s}$ is the solvent viscosity.

$\eta_{\mathrm{sp}}=\frac{\eta_{0}-\eta_{\mathrm{s}}}{\eta_{\mathrm{s}}}$

Results presented in Table 2 show the same trend as seen in the zeroshear rate viscosity measurements. The values increase for higher polymer concentrations and reach higher values for HPMC HMW when compared to HPMC LMW. Results also show that at the highest concentrations of HPMC LMW (6\%) the values are similar to those found for the lowest concentration of the HPMC HMW (1\%). This indicates that at the highest concentrations of HPMC LMW the EHD processing could result in a structure of particles with fibres (or fibres with particles), something that was confirmed in the SEM analysis presented below.

In the dilute regime $\left(C \ll C_{\mathrm{e}}\right)$, the specific viscosity $\left(\eta_{s p}\right)$ increases exponentially with concentration, presenting a slope of $1.0\left(C^{1.0}\right)$, while in the semi-dilute unentangled regime $\left(C<C_{\mathrm{e}}\right)$ the exponential increase presents a slope value of $1.25\left(C^{1.25}\right)$. In these regimes, the formation of particles and beaded fibres is favoured, but as concentration increases, chain overlapping initiates, viscosity changes abruptly, and a point, that can be defined as the entanglement concentration $\left(C=C_{\mathrm{e}}\right), C_{\mathrm{e}}$ is reached. A semi-diluted entangled regime is entered $\left(C>C_{\mathrm{e}}\right)$ and $\eta_{s p}$ reflects the abrupt change in viscosity by scaling with concentration to the power of $4.8\left(C^{4.8}\right)$. A concentrated regime $\left(C^{* *}\right)$ is entered as concentration and viscosity further increase and $\eta_{s p}$ scales at the power of $3.6\left(C^{3.6}\right)$. To determine the solution type of regime, as well as to estimate $C_{\mathrm{e}}$ and $C^{* * *}$, specific viscosity and concentration were plotted in a log-log plot. These plots are presented in Fig. 2. The abrupt changes in the slope mark the transition between different regimes. $C_{\mathrm{e}}$ marks the beginning of the semi diluted entangled regime, and $C^{* *}$ marks the beginning of the concentrated regime, both of which are regions more prone to produce fibres, with the concentrated regime typically producing smoother fibres with lower diameters (Bock et al., 2012; Lee et al., 2018; McKee et al., 2004; Shenoy et al., 2005).

For the HPMC HMW, the first slope change occurs when the concentration moves from 1 to $1.5 \%$, marking the transition from the semi dilute unentangled regime to the semi dilute entangled regime, in which the $C_{\mathrm{e}}$ is determined to be $1.5 \%$. The second slope change (as concentration increases from $1.5 \%$ to $2 \%)$ marks the concentrated regime $\left(C^{* *}\right.$ $=2 \%$ ). Slope changes displayed in Fig. 2 show that the semi-dilute entangled regime, $\eta_{s p} \sim C^{4.61}$, and the concentrated regime, $\eta_{s p} \sim$ $C^{3.88}$, are in good agreement with the theoretically predicted scaling law exponents' values of 4.8 and 3.8. The dilute and semi-dilute unentangled regimes were not determined as rheological analysis was not conducted below $1 \%$. Results would indicate that an electrospinning zone can be found for concentrations above $1.5 \%$, namely around $2 \%$ where the $C^{* *}$ was found.

Regarding the HPMC LMW, the first slope change occurs when the concentration increases from $1 \%$ to $5 \%$, with an $\eta_{s p} \sim C^{2.77}$, while the 


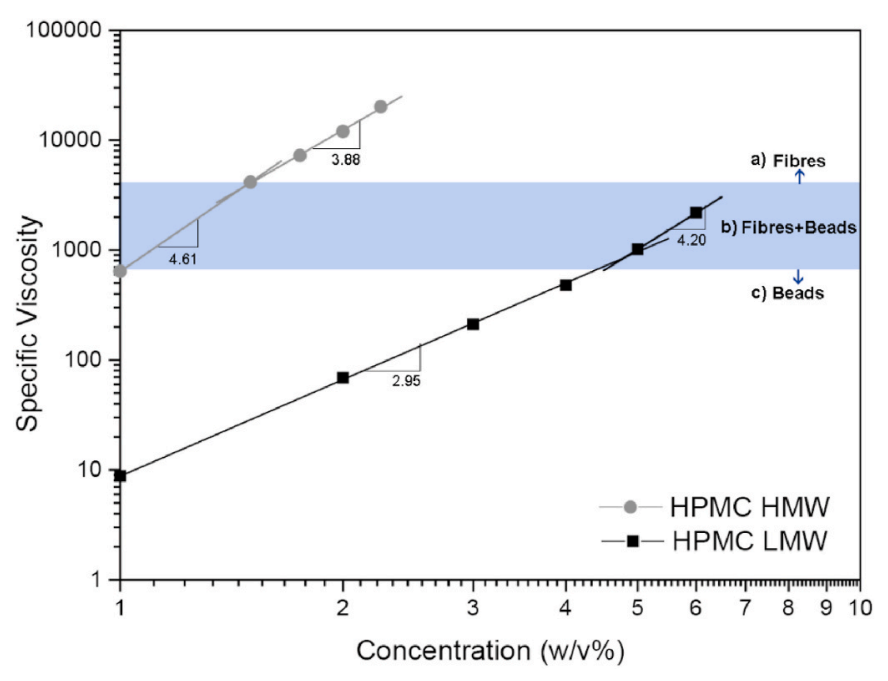

Fig. 2. Specific viscosity for different concentrations of HPMC LMW (Mw $=90$ $\mathrm{kDa})$ and HPMC HMW (Mw $=746 \mathrm{kDa})$. The blue zone represents the conditions where fibre + beads are obtained. (For interpretation of the references to colour in this figure legend, the reader is referred to the Web version of this article.) second slope change occurs between $5 \%$ and $6 \%$, with an $\eta_{s p} \sim C^{4.20}$. It marks the transition between the semi-diluted unentangled regime and the semi-diluted entangled regime, in which $C_{\mathrm{e}}=6 \%$, shifting from a zone in which the formation of particles is expected to a zone in which fibres would be the obtained structure. Results show that for the semidiluted unentangled regime the obtained values $\left(\eta_{\mathrm{sp}} \sim C^{2.95}\right)$ do not present a good fit with the theorical values $\left(\eta_{s p} \sim C^{1.25}\right)$, however for the semi dilute entangled regime, the obtained values $\eta_{s p} \sim C^{4.8}$, fit well with the theoretical value $\left(\eta_{s p} \sim C^{4.20}\right)$.

This crossover point is usually identified as ideal to produce particles, as there is enough entanglement of molecular chains in this transition to produce particles in a stable and reproducible form, without producing fibres. Nevertheless, transition zones can be a fertile ground for the production of mixed structures (with both particles and fibres) as slight changes in the EHD parameters (e.g., voltage, flow rate, working distance) will influence the final outcome (Bock et al., 2012; Faramarzi et al., 2016; Lee et al., 2018; Shenoy et al., 2005). After the determination of theoretical electrospraying and electrospinning zones, the structures obtained from the polymer solutions processed by EHD were evaluated regarding their morphology.

\subsection{Micro and nano structure analysis}

The electrospraying and electrospinning zones that were previously determined, were confirmed by submitting the polymer solutions to EHD processing. Needle diameter, solution flow rate, voltage and

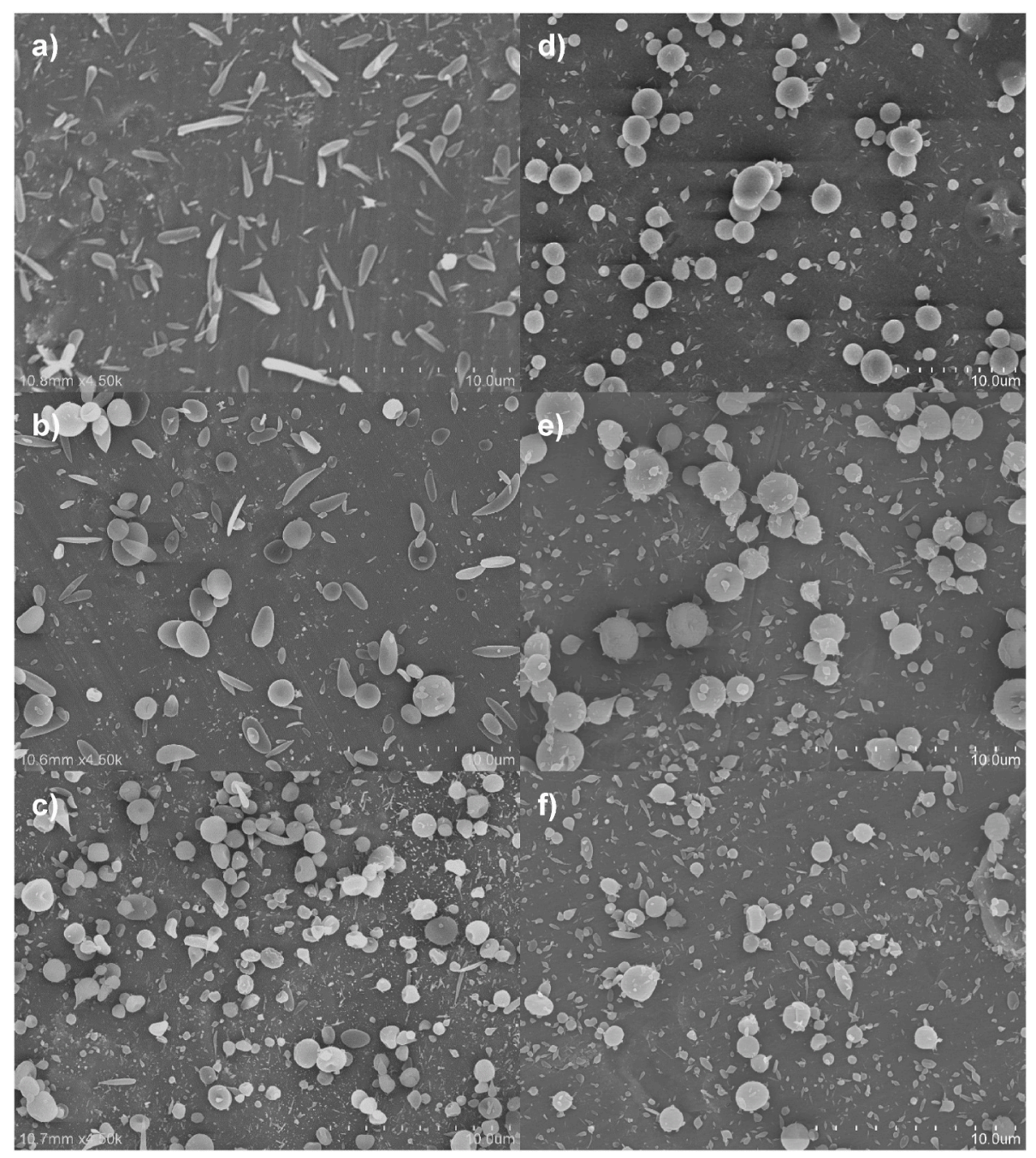

Fig. 3. SEM imaging for the low viscosity samples. a) $1 \%$, b) $2 \%$, c) $3 \%$, d) $4 \%$, e) $5 \%$, f) $6 \%$. 
distance values were kept constant, except for one formulation of HPMC HMW in which voltage varied and was adjusted to obtain a visible Taylor cone at the tip of the spinneret and without visible droplets in the collector. Typically, for higher concentrations, due to higher sample viscosity, higher voltage and distance combinations were needed to produce a stable Taylor cone. The resulting structures were evaluated by SEM and are presented in Fig. 3. It is possible to see that for all tested concentrations some type of structure was produced. At low concentrations, namely 1 and $2 \%(\mathrm{w} / \mathrm{v})$, rod-shaped structures were formed, but by increasing the concentration (up to $5 \%$ ) rounder particles were produced and at the highest tested concentration (6\%) larger beads connected with fibres, 'beads-on-a-string', were obtained. This was more noticeable at higher flow rates than lower flow rates.

Based on the results, low concentrations of HPMC LMW (1-2\%) were not considered as appropriate for electrospraying, as round particles were not obtained, while the highest concentration (6\%) was also not considered appropriate due to the simultaneous production of round particles and fibres. These results agree with the results obtained in the specific viscosity versus concentration plot analysis, where an abrupt increase in viscosity is seen at $6 \%(\mathrm{w} / \mathrm{v})$ solutions, indicating the presence of both beads and fibres. Thus, based on these results, polymer concentrations between 3 and $5 \%(\mathrm{w} / \mathrm{v})$ seem more appropriate to produce particles.

Fig. 4 presents SEM images of the structures obtained using HPMC HMW. Results show that HPMC HMW was appropriate to produce fibres as all tested formulations resulted in fibres, although in some of the formulations used (namely at the lower concentrations of 1 and 1.5\%) beaded fibres were obtained. It was also observed that some samples presented droplets among the fibres, indicating that higher voltages might be needed for appropriate processing, namely, to allow a proper solvent evaporation. Less beading of the fibres was observed at higher polymer concentrations (from 1.75 to $2.25 \% \mathrm{w} / \mathrm{v}$ ) which seem to be more appropriate to obtain fibres. As observed for the particle production, the electrospinning zones predicted through the specific viscosity versus concentration plot analyses matched the results observed by SEM, given that samples above $1.5 \%$ seemed to produce more adequate fibres, with less beading.

The structures observed in Figs. 3 and 4 show the adequacy of the previously predicted electrospraying and electrospinning zones for both HPMC tested (LMW and HMW), validating the use of this methodology for HPMC. In Fig. 5 it is also possible to see the influence of other parameters of EHD processing, especially near transition zones, on the structural morphology (in this case, the influence of flow rate). In one of those regime's transition zones, at a concentration of $6 \%$, an increase of the amount of fibres in the 'beads-on-string' structure can be obtained by changing the flow rate of the EHD process from 500 to $1000 \mu \mathrm{L} / \mathrm{h}$.

Fig. 5 shows that despite being extremely important variables, polymer concentration and viscosity are not be-alls and end-alls when it comes to determining and tuning the structure morphology, and a more comprehensive study of the parameters that influence structure morphology, and how they influence it, is necessary. As such, further research on the influence of processing and solution parameters or

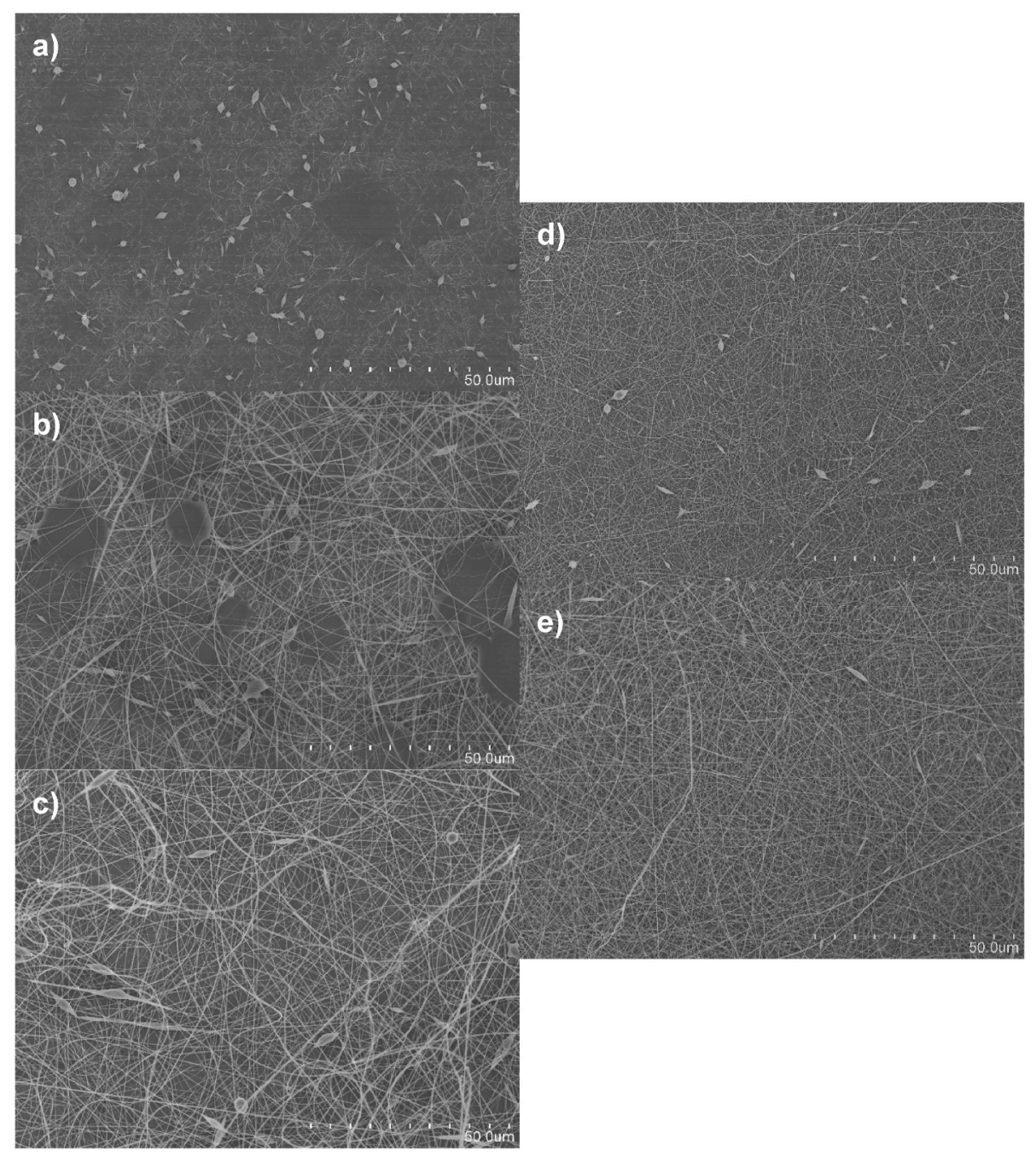

Fig. 4. SEM imaging for the high viscosity samples. a) $1 \%$, b) $1.5 \%$, c) $1.75 \%$, d) $2 \%$, e) $2.25 \%$. 


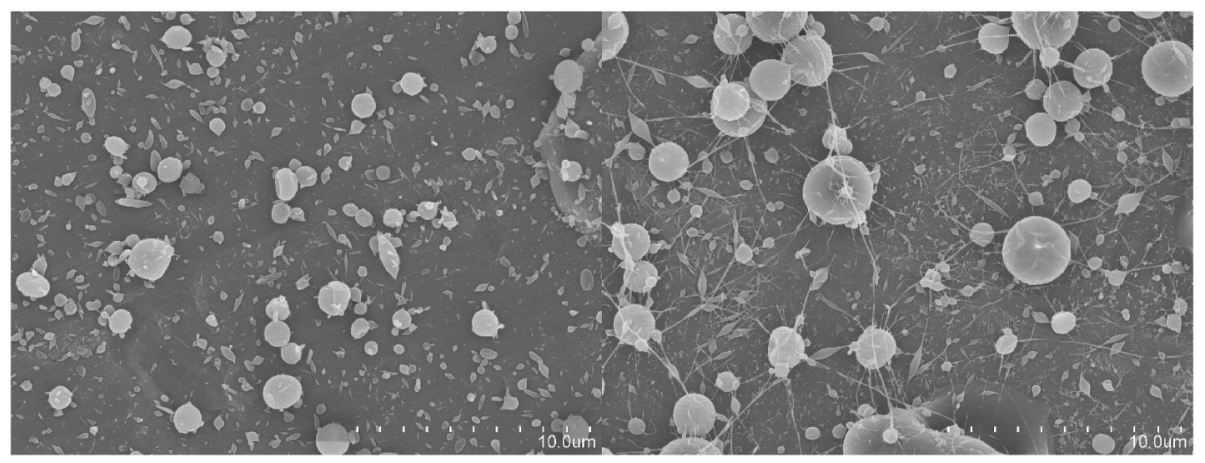

Fig. 5. Comparison between microstructures produced at different flow rates. $500 \mu \mathrm{L} / \mathrm{h}$ (left), $1000 \mu \mathrm{L} / \mathrm{h}$ (right). Remaining parameters were the same. Polymer concentration of $6 \%(\mathrm{w} / \mathrm{v})$ of HPMC LMW, voltage $10 \mathrm{kV}$, distance $15 \mathrm{~cm}$.

properties should be conducted.

Sample morphology was analysed, and structures produced from HPMC LMW displayed rod-like and spherical morphology, and in some formulations produce both particles and fibres. HPMC HMW produced mostly fibrous structures, at times accompanied by particles. HPMC LMW structures were characterised regarding their particle diameter and sphericity, while HPMC HMW were characterised regarding their fibre diameter. These properties are presented in Table 3 and showed that microparticles size ranged between 800 and $1200 \mathrm{~nm}$. When low concentrations are used ( 1 and $2 \%$ ) produced structures displayed a rod like morphology, while when higher concentrations are used (between 3 and $6 \%$ ) the process produced more spherical particles. For microparticles produced using 3 and 4\% of HPMC the aspect ratio (the closer the aspect ratio is to one the more spherical the particles are) is closer to one than that the microparticles produced at 5 and $6 \%$, formulations in

Table 3

Aspect Ratio, aspect ratio uniformity, diameter, diameter uniformity and span of LMW and HMW HPMC micro and nanostructures.

\begin{tabular}{|c|c|c|c|c|c|}
\hline Sample & $\begin{array}{l}\text { Aspect } \\
\text { Ratio }\end{array}$ & $\begin{array}{l}\text { Aspect Ratio } \\
\text { Uniformity }\end{array}$ & $\begin{array}{l}\text { Diameter } \\
(\mathrm{nm})\end{array}$ & $\begin{array}{l}\text { Diameter } \\
\text { Uniformity }\end{array}$ & Span \\
\hline $\begin{array}{l}\text { LMW } \\
\qquad \begin{array}{l}\text { HPMC } \\
1 \%\end{array}\end{array}$ & 3.7 & 0.15 & 973 & 0.52 & 2.38 \\
\hline $\begin{array}{l}\text { LMW } \\
\text { HPMC } \\
2 \%\end{array}$ & 2.1 & 0.27 & 994 & 0.25 & 1.31 \\
\hline $\begin{array}{l}\text { LMW } \\
\text { HPMC } \\
3 \%\end{array}$ & 1.3 & 0.23 & 833 & 0.20 & 1.18 \\
\hline $\begin{array}{l}\text { LMW } \\
\text { HPMC } \\
4 \%\end{array}$ & 1.3 & 0.10 & 1188 & 0.28 & 1.65 \\
\hline $\begin{array}{l}\text { LMW } \\
\text { HPMC } \\
5 \%\end{array}$ & 2.0 & 0.48 & 906 & 0.32 & 1.74 \\
\hline $\begin{array}{l}\text { LMW } \\
\text { HPMC } \\
6 \%\end{array}$ & 1.7 & 0.54 & 931 & 0.43 & 1.79 \\
\hline $\begin{array}{l}\text { HMW } \\
\text { HPMC } \\
1 \%\end{array}$ & - & - & 151 & 1.93 & 1.59 \\
\hline $\begin{array}{l}\text { HMW } \\
\text { HPMC } \\
1.5 \%\end{array}$ & - & - & 142 & 0.15 & 0.90 \\
\hline $\begin{array}{l}\text { HMW } \\
\text { HPMC } \\
1.75 \%\end{array}$ & - & - & 161 & 0.34 & 0.88 \\
\hline $\begin{array}{l}\text { HMW } \\
\text { HPMC } \\
2 \%\end{array}$ & - & - & 79 & 0.14 & 0.82 \\
\hline $\begin{array}{l}\text { HMW } \\
\text { HPMC } \\
2.25 \%\end{array}$ & - & - & 107 & 0.12 & 0.81 \\
\hline
\end{tabular}

which fibres were also produced. Formulations with 3 and $4 \%$ of HPMC also lead to good aspect ratio uniformity, diameter uniformity and span values. Particle diameters obtained here agree well with the results reported by Huang et al. (2019), in which a modified coaxial electrospray was performed, using HPMC with the same type of substitution (type E, methoxyl 28-30\%, hydroxypropyl 7-12\%), a lower viscosity (5 mPa s) and using a mixture of ethanol and dichloromethane (1:1) as solvent. The process was carried out at lower flow rates, which has been reported to decrease size distribution, but also has the drawback of decreasing the productivity (Faramarzi et al., 2016; Lee et al., 2018).

The produced nanofibres displayed mean diameters ranging from 80 to $160 \mathrm{~nm}$, with mostly low diameter uniformity and span values. The lowest diameter uniformity and span values were obtained at a concentration of $2.25 \%$, respectively with values of 0.121 and 0.805 . The highest values of diameter uniformity and span were obtained at a concentration of $1 \%$, respectively 1.93 and 1.59 . This is probably because in this formulation beaded fibres were produced, causing a lower uniformity of the produced structures. An overarching trend regarding the influence of concentration on fibre diameter does not seem to exist, possibly due to the presence of beaded fibres in some of the samples, which artificially increase their mean diameter. For samples without beaded fibres (concentration at $2 \%$ and $2.25 \%$ ) an increase in concentration leads to higher fibre diameter. In another work, aiming to produce nanofibres of similar diameters (around $120 \mathrm{~nm}$ ) higher concentrations of HPMC and higher voltages where needed. This might be due to the use of slightly different processing parameters, namely the use of a different ratio (1:1) of water-ethanol, which resulted in lower viscosities and higher surface tensions (Frenot, Henriksson, \& Walkenström, 2007).

\section{Conclusions}

The use of EHD processing allowed to produce micro and nano structures using low and high viscosity HPMC. Round and spherical particles were produced using the HPMC LMW, at concentrations ranging between 3 and $5 \%(w / v)$. All tested formulations of HPMC HMW were able to produce fibres, while the use of lower concentrations of HPMC HMW led to the production of beaded fibres (between 1 and $1.5 \%)$, while higher concentrations (1.75-2.25\%) led to the production of less beaded and smoother fibres. SEM morphology results confirmed the information obtained from the specific viscosity versus concentration plots for electrospraying and electrospinning zones. An electrospraying zone was found between $1 \%$ and $5 \%$ of HPMC LMW, while at $6 \%$ particles of larger diameters and a mix of particles and fibres started to form, indicating a transition to an electrospinning zone. Particle diameter varied between 800 and $1200 \mathrm{~nm}$, while aspect ratio varied between 1.316 and 3.732 (spherical particles and rod-shaped structures, respectively). Regarding HPMC HMW, fibres were formed at all tested concentrations, but at lower concentrations (below 1.5\%) beaded fibres 
were produced. An electrospinning zone was thus defined between $1.5 \%$ and $2.25 \%$, with higher concentrations producing smoother fibres. The mean fibre diameter ranged from 80 to $160 \mathrm{~nm}$. Additionally, it was also possible to demonstrate that for systems operating near transition zones, other parameters such as the flow rate, can have a great influence on the structure morphology. Therefore, future work should focus on the assessment of the influence of the remaining parameters on particle and fibre morphology, especially doing so at higher flow rates to increase productivity as this is one of the main drawbacks of the use of EHD processing. This assessment would ideally be conducted with a Design of Experiments methodology to understand better the relationship and influence between the different process or solution parameters and the morphological outcome of the produced structures.

\section{Author statement}

Conceptualization, P.M.S., C.P., J.M.L., L.M.P., M.A.C., A.A.V. and M.C.; methodology, P.M.S., C.P., M.C.; investigation, P.M.S., C.P., J.M. L., L.M.P., M.A.C., A.A.V. and M.C.; formal analysis, P.M.S.; writing—original draft preparation, P.M.S.; writing—review and editing, P. M.S., C.P., J.M.L., L.M.P., M.A.C., A.A.V. and M.C.; supervision, C.P., J. M.L., L.M.P., M.A.C., A.A.V. and M.C. All authors have read and agreed to the published version of the manuscript.

\section{Declaration of competing interest}

The authors declare that they have no known competing financial interests or personal relationships that could have appeared to influence the work reported in this paper.

\section{Acknowledgments}

We would like to acknowledge the European Commission through the H2020-MSCA-RISE project FODIAC-Food for Diabetes and Cognition (reference number 778388), the Portuguese Foundation for Science and Technology (FCT) under the scope of the strategic funding of UID/ BIO/04469/2020 unit and BioTecNorte operation (NORTE-01-0145FEDER-000004) funded by the European Regional Development Fund under the scope of Norte2020 - Programa Operacional Regional do Norte. The author Pedro Silva is the recipient of a fellowship (SFRD/BD/ 130247/2017) supported by Fundação para a Ciência e a Tecnologia, (FCT, Portugal).

\section{References}

Abuzar, S. M., Hyun, S. M., Kim, J. H., Park, H. J., Kim, M. S., Park, J. S., et al. (2018) Enhancing the solubility and bioavailability of poorly water-soluble drugs using supercritical antisolvent (SAS) process. International Journal of Pharmaceutics, 538 (1-2), 1-13. https://doi.org/10.1016/j.ijpharm.2017.12.041

Aydogdu, A., Sumnu, G., \& Sahin, S. (2019). Fabrication of gallic acid loaded Hydroxypropyl methylcellulose nanofibers by electrospinning technique as active packaging material. Carbohydrate Polymers, 208, 241-250. https://doi.org/10.1016/ j.carbpol.2018.12.065

Bhushani, J. A., Kurrey, N. K., \& Anandharamakrishnan, C. (2017). Nanoencapsulation of green tea catechins by electrospraying technique and its effect on controlled release and in-vitro permeability. Journal of Food Engineering, 199, 82-92. https://doi.org/ 10.1016/j.jfoodeng.2016.12.010

Bock, N., Dargaville, T. R., \& Woodruff, M. A. (2012). Electrospraying of polymers with therapeutic molecules: State of the art. Progress in Polymer Science, 37(11), 1510-1551. https://doi.org/10.1016/j.progpolymsci.2012.03.002

Bourbon, A. I., Barbosa-Pereira, L., Vicente, A. A., Cerqueira, M. A., \& Pastrana, L. (2020). Dehydration of protein lactoferrin-glycomacropeptide nanohydrogels. Food Hydrocolloids, 101, Article 105550. https://doi.org/10.1016/j. foodhyd.2019.105550

Burdock, G. A. (2007, December 1). Safety assessment of hydroxypropyl methylcellulose as a food ingredient. Food and Chemical Toxicology (Vol. 45,, 2341-2351. https://doi. org/10.1016/j.fct.2007.07.011

Cerqueira, M. A., Pinheiro, A. C., Silva, H. D., Ramos, P. E., Azevedo, M. A., FloresLópez, M. L., et al. (2014). Design of bio-nanosystems for oral delivery of functional compounds. Food Engineering Reviews, 6(1-2), 1-19. https://doi.org/10.1007/ s12393-013-9074-3
Chowhan, Z. T. (1980). Role of binders in moisture-induced hardness increase in compressed tablets and its effect on in vitro disintegration and dissolution. Journal of Pharmaceutical Sciences, 69(1), 1-4. https://doi.org/10.1002/jps.2600690102

Costa, M. J., Ramos, P. E., Fuciños, P., Teixeira, J. A., Pastrana, L. M., \& Cerqueira, M.Â. (2019). Development of bio-based nanostructured systems by electrohydrodynamic processes. In Nanotechnology applications in the food industry (pp. 3-20). https://doi. org/10.1201/9780429488870-1

Deng, L., Kang, X., Liu, Y., Feng, F., \& Zhang, H. (2018). Characterization of gelatin/zein films fabricated by electrospinning vs solvent casting. Food Hydrocolloids, 74, 324-332. https://doi.org/10.1016/j.foodhyd.2017.08.023

Faramarzi, A. R., Barzin, J., \& Mobedi, H. (2016). Effect of solution and apparatus parameters on the morphology and size of electrosprayed PLGA microparticles. Fibers and Polymers, 17(11), 1806-1819. https://doi.org/10.1007/s12221-0166685-3

Frenot, A., Henriksson, M. W., \& Walkenström, P. (2007). Electrospinning of cellulosebased nanofibers. Journal of Applied Polymer Science, 103(3), 1473-1482. https://doi. org/10.1002/app.24912

García-Moreno, P. J., Mendes, A. C., Jacobsen, C., \& Chronakis, I. S. (2018). Biopolymers for the nanomicroencapsulation of bioactive ingredients by electrohydrodynamic processing. In Polymers for food applications (pp. 447-479). https://doi.org/10.1007/ 978-3-319-94625-2_17

Huang, W., Hou, Y., Lu, X., Gong, Z., Yang, Y., Lu, X. J., et al. (2019). The process-property-performance relationship of medicated nanoparticles prepared by modified coaxial electrospraying. Pharmaceutics, 11(5), 226. https://doi.org/ 10.3390/pharmaceutics11050226

Karim, F. T., Sarker, Z. M., Ghafoor, K., Al-Juhaimi, F. Y., Jalil, R., Awang, M. B., et al. (2016). Microencapsulation of fish oil using hydroxypropyl methylcellulose as a carrier material by spray drying. Journal of Food Processing and Preservation, 40(2), 140-153. https://doi.org/10.1111/jfpp.12591

Katona, J. M., Sovilj, V. J., Petrović, L. B., \& Milanović, J. L. (2010). Preparation and characterization of oil containing microcapsules obtained by an interaction induced coacervation. Journal of Dispersion Science and Technology, 31(12), 1679-1684. https://doi.org/10.1080/01932690903297231

Kaur, G., Grewal, J., Jyoti, K., Jain, U. K., Chandra, R., \& Madan, J. (2018). Oral controlled and sustained drug delivery systems: Concepts, advances, preclinical, and clinical status. In Drug targeting and stimuli sensitive drug delivery systems (pp. 567-626). https://doi.org/10.1016/B978-0-12-813689-8.00015-X

Lasprilla-Botero, J., Álvarez-Láinez, M., \& Lagaron, J. M. (2018). The influence of electrospinning parameters and solvent selection on the morphology and diameter of polyimide nanofibers. Materials Today Communications, 14, 1-9. https://doi.org/ 10.1016/j.mtcomm.2017.12.003

Lee, H., An, S., Kim, S., Jeon, B., Kim, M., \& Kim, I. S. (2018). Readily functionalizable and stabilizable polymeric particles with controlled size and morphology by electrospray. Scientific Reports, 8(1), 1-10. https://doi.org/10.1038/s41598-01834124-0

Liao, Y., Loh, C. H., Tian, M., Wang, R., \& Fane, A. G. (2018). Progress in electrospun polymeric nanofibrous membranes for water treatment: Fabrication, modification and applications. Progress in Polymer Science, 77, 69-94. https://doi.org/10.1016/j. progpolymsci.2017.10.003

Limongi, T., Tirinato, L., Pagliari, F., Giugni, A., Allione, M., Perozziello, G., et al. (2017). January 1). Fabrication and applications of micro/nanostructured devices for tissue engineering. Nano-Micro Letters (Vol. 9,, 1-13. https://doi.org/10.1007/s40820016-0103-7

Mahesh, B., Kathyayani, D., Nanjundaswamy, G. S., Channe Gowda, D., \& Sridhar, R. (2019). Miscibility studies of plastic-mimetic polypeptide with hydroxypropylmethylcellulose blends and generation of non-woven fabrics. Carbohydrate Polymers, 212, 129-141. https://doi.org/10.1016/j. carbpol.2019.02.042

Marques, A. M., Azevedo, M. A., Teixeira, J. A., Pastrana, L. M., Gonçalves, C., \& Cerqueira, M. A. (2019). Engineered nanostructures for enrichment and fortification of foods. In Food applications of nanotechnology (pp. 61-97). https://doi.org/ 10.1201/9780429297038-4

McKee, M. G., Wilkes, G. L., Colby, R. H., \& Long, T. E. (2004). Correlations of solution rheology with electrospun fiber formation of linear and branched polyesters. Macromolecules, 37(5), 1760-1767. https://doi.org/10.1021/ma035689h

Nunes, R., Pereira, B. D., Cerqueira, M., Silva, P. M., Pastrana, L., Vicente, A. A., et al. (2020). Lactoferrin-based nanoemulsions to improve the physical and chemical stability of omega-3 fatty acids. Food \& Function, 11(3), 1966-1981. https://doi.org/ 10.1039/c9fo02307k

Pal, K., Paulson, A. T., \& Rousseau, D. (2013). Biopolymers in controlled-release delivery systems. In Handbook of biopolymers and biodegradable plastics: Properties, processing and applications (pp. 329-363). https://doi.org/10.1016/B978-1-4557-28343.00014-8

Rodrigues, R. M., Ramos, P. E., Cerqueira, M. F., Teixeira, J. A., Vicente, A. A., Pastrana, L. M., et al. (2020). Electrosprayed whey protein-based nanocapsules for $\beta$-carotene encapsulation. Food Chemistry, 314, Article 126157. https://doi.org/ 10.1016/j.foodchem.2019.126157

Romita, D., Cheng, Y. L., \& Diosady, L. L. (2011). Microencapsulation of ferrous fumarate for the production of salt double fortified with iron and iodine. International Journal of Food Engineering, 7(3). https://doi.org/10.2202/1556-3758.2122

Senthil Muthu Kumar, T., Senthil Kumar, K., Rajini, N., Siengchin, S., Ayrilmis, N., \& Varada Rajulu, A. (2019). A comprehensive review of electrospun nanofibers: Food and packaging perspective. Composites Part B: Engineering, 175, Article 107074. https://doi.org/10.1016/j.compositesb.2019.107074

Shenoy, S. L., Bates, W. D., Frisch, H. L., \& Wnek, G. E. (2005). Role of chain entanglements on fiber formation during electrospinning of polymer solutions: Good 
solvent, non-specific polymer-polymer interaction limit. Polymer, 46(10), 3372-3384. https://doi.org/10.1016/j.polymer.2005.03.011

Silva, H. D., Beldíková, E., Poejo, J., Abrunhosa, L., Serra, A. T., Duarte, C. M. M., et al. (2019). Evaluating the effect of chitosan layer on bioaccessibility and cellular uptake of curcumin nanoemulsions. Journal of Food Engineering, 243, 89-100. https://doi. org/10.1016/j.jfoodeng.2018.09.007

Smeets, A., Clasen, C., \& Van den Mooter, G. (2017). Electrospraying of polymer solutions: Study of formulation and process parameters. European Journal of Pharmaceutics and Biopharmaceutics, 119, 114-124. https://doi.org/10.1016/j. ejpb.2017.06.010

Smeets, A., Koekoekx, R., Clasen, C., \& Van den Mooter, G. (2018). Amorphous solid dispersions of darunavir: Comparison between spray drying and electrospraying. European Journal of Pharmaceutics and Biopharmaceutics, 130, 96-107. https://doi. org/10.1016/j.ejpb.2018.06.021

Stephen, A. M., Phillips, G. O., \& Williams, P. A. (2016). Food polysaccharides and their applications: Second edition. In Food polysaccharides and their applications: Second edition.

Sun, G., Liang, T., Tan, W., \& Wang, L. (2018). Rheological behaviors and physical properties of plasticized hydrogel films developed from $\kappa$-carrageenan incorporating hydroxypropyl methylcellulose. Food Hydrocolloids, 85, 61-68. https://doi.org/ 10.1016/j.foodhyd.2018.07.002

Tanti, R., Barbut, S., \& Marangoni, A. G. (2016). Hydroxypropyl methylcellulose and methylcellulose structured oil as a replacement for shortening in sandwich cookie creams. Food Hydrocolloids, 61, 329-337. https://doi.org/10.1016/j. foodhyd.2016.05.032

Tiwari, S. K., \& Venkatraman, S. S. (2012). Importance of viscosity parameters in electrospinning: Of monolithic and core-shell fibers. Materials Science and Engineering: C, 32(5), 1037-1042. https://doi.org/10.1016/j.msec.2012.02.019

Wang, J., Jansen, J. A., \& Yang, F. (2019, April 25). Electrospraying: Possibilities and challenges of engineering carriers for biomedical applications - a mini review. Frontiers in Chemistry (Vol. 7,, 258. https://doi.org/10.3389/fchem.2019.00258

Zhang, F., Ni, Q., Jacobson, O., Cheng, S., Liao, A., Wang, Z., et al. (2018). Polymeric nanoparticles with a glutathione-sensitive heterodimeric multifunctional prodrug for in vivo drug monitoring and synergistic cancer therapy. Angewandte Chemie

International Edition, 57(24), 7066-7070. https://doi.org/10.1002/anie.201801984.

Zhou, H., Shi, Z., Wan, X., Fang, H., Yu, D. G., Chen, X., et al. (2019). The relationships between process parameters and polymeric nanofibers fabricated using a modified coaxial electrospinning. Nanomaterials, 9(6), 843. https://doi.org/10.3390/ nano9060843 